\title{
Orthogeriatrics: hip fracture and its implications
}

\author{
Heinrich Wolfgang Thaler · Markus Gosch · Christian Kammerlander
}

Received: 18 June 2013 / Accepted: 12 July 2013 / Published online: 16 August 2013

(C) Springer-Verlag Wien 2013

As the aging population grows rapidly, the number of patients with geriatric fractures increases on an even higher rate. Adults over 85 years old have an approximately ten times higher risk to sustain a hip fracture than those aged 65-70 years [1]. The cause for most of these "fragility fractures" is a simple fall, a calamity to which older people are predisposed by cardiovascular disease, neurological disorders, frailty, polypharmacy, and many other geriatric syndromes. Hip fracture is a typical low trauma fracture, occurring mostly in people with osteoporosis. It can be considered as a prototypical geriatric illness and it is a potentially devastating condition. The imminent treatment of proximal femoral fracture consists of major orthopedic surgery in most cases. In spite of the very effective and worldwide wellestablished surgical procedures (total or partial hip arthroplasty, osteosynthesis), hip fracture frequently leads to pain, immobilization, and a plethora of complications like thromboembolism, infection, gastrointestinal bleeding, delirium, and functional loss. Due to these circumstances, the treatment course after hip fracture is frequently prolonged, the recovery is uncertain and the outcome often leads to functional decline, institutionalization, and death. Therefore, extensive support including many disciplines is required. Comprehensive peri- and postoperative care is very important in this context, but understanding geriatric principles is also a

H. W. Thaler $(\bowtie)$

Unfallkrankenhaus/Trauma Center Meidling,

Kundratstrasse 37, 1120 Vienna, Austria

e-mail: heinrich.thaler@auva.at

M. Gosch

Krankenhaus Hochzirl, Zirl, Austria

C. Kammerlander

Medical University Innsbruck, Innsbruck, Austria conditio sine qua non for all who look after patients with hip fractures.

The outcome for older patients with hip fractures is poor. Approximately $25 \%$ of older patients who sustain hip fractures die within 1 year and about $13 \%$ die within 6 months after the trauma [2]. Also the recovery rate among those who survived for 6 months is poor: only $50 \%$ recover their ability to perform activities of daily living (ADLs) as prior to the fracture [3]. After a proximal femoral fracture, older patients are five times more likely to be institutionalized than age-matched controls without trauma [4].

It should always be kept in mind that the primary objective of hip fracture surgery is to return patients to the level of functioning prior to the trauma. Therefore it is important, particularly for patients with life-limiting diseases (bedridden patients, advanced dementia), that the goals of care are determined as soon as possible. The surgical risk should always be considered in the light of the patient needs and a discussion whether nonoperative management may be more appropriate is frequently necessary. On the other hand, a surgical delay of more than 2 days from the time of admission doubles the risk of 1-year mortality; the delay is mostly a consequence of the comorbid conditions [5].

Preoperative care includes pain control, correcting medical abnormalities, and considering timing and type of surgical procedure as well as anesthetic techniques.

Perioperatively, a mainstay of treatment is medical thromboprophylaxis, chemoprophylaxis with antibiotics, delirium prevention, stress bleeding prophylaxis, the correction of major clinical abnormalities, and pain control. The best way to incorporate these multidisciplinary care elements, which can significantly decrease complications during hospital stay, is to involve a geriatrician or an internist [6], as shown in an exemplary orthogeriatric comanagement model like the Tyrolean geriatric fracture center [7]. Early mobilization (if possible starting on 
the next morning after surgery) is also very important, it improves functional recovery and mortality [8].

As the patient with hip fracture passes through many institutions (acute trauma surgery, remob/acute geriatrics, rehabilitation, home) a lot of important information can get lost at these vulnerable points on the way to recovery. Particularly secondary prevention of subsequent fractures is often overlooked because it is not a part of the acute care management in most countries. If osteoporosis has not previously been diagnosed, vitamin D and calcium supplementation should be started immediately after surgery [9] and a specific medical treatment for osteoporosis (e.g., with bisphosphonates) should be initiated within 90 days after surgery [10]. To improve recovery and to maximize restitution, rehabilitation exercises in institutions, at home or in groups should be initiated. Physicians should be able to anticipate problems that arise during the recovery process. Rehabilitation strategies must be tailored to patient needs and overall goals should be determined by a team of dedicated professionals to optimize outcomes.

In this special issue of $W M W$, the most important topics concerning hip fracture and its complications are presented by the working group on orthogeriatrics of the Austrian Society on Geriatrics and Gerontology. The articles are the results of thorough data collecting, frequent discussions, and several adjustment meetings of the team, composed of dedicated geriatricians who work as trauma surgeons, internists, anesthesists, and nurses. This multidisciplinary approach is of special importance, because our common objective is to improve outcomes and to maximize recovery in this rapidly growing population of high-risk geriatric patients.

\section{Acknowledgements}

We are very grateful to Simon Pienaar, Trauma Center Meidling, for his help in preparing this special issue.

\section{Conflict of interest}

The authors declare that they have no conflict of interest.

\section{References}

1. Samelson EJ, Zhang Y, Kiel DP, et al. Effect of birth cohort on risk of hip fracture: age-specific incidence rates in the Framingham Study. Am J Public Health. 2002 May;92(5):858-62.

2. Hannan EL, Magaziner J, Wang J, et al. Mortality and locomotion 6 months after hospitalization for hip fracture: risk factors and risk-adjusted hospital outcomes. JAMA. 2001 Jun 6;285(21):2736-42.

3. Magaziner J, Simonsick EM, Kashner TM, et al. Predictors of functional recovery one year following hospital discharge for hip fracture: a prospective study. J Gerontol. 1990 May;45(3):M101-7.

4. Penrod JD, Litke A, Hawkes WG, et al. Heterogeneity in hip fracture patients: age, functional status, and comorbidity. J Am Geriatr Soc. 2007 Mar;55(3):407-13.

5. Zuckerman JD, Skovron ML, Koval KJ, et al. Postoperative complications and mortality associated with operative delay in older patients who have a fracture of the hip. J Bone Joint Surg Am. 1995 Oct;77(10):1551-6.

6. Pedersen SJ, Borgbjerg FM, Schousboe B, Hip Fracture Group of Bispebjerg Hospital. A comprehensive hip fracture program reduces complication rates and mortality. J Am Geriatr Soc. 2008 Oct;56(10):1831-8.

7. Kammerlander C, Gosch M, Blauth M, et al. The Tyrolean Geriatric Fracture Center. an orthogeriatric co-management model.Z Gerontol Geriatr. 2011 Dec;44(6):363-7.

8. Siu AL, Penrod JD, Boockvar KS, et al. Early ambulation after hip fracture: effects on function and mortality. Arch Intern Med. 2006 Apr 10;166(7):766-71.

9. Grant AM, Avenell A, Campbell MK, et al. RECORD Trial Group. Oral vitamin D3 and calcium for secondary prevention of low-trauma fractures in elderly people (Randomised Evaluation of Calcium Or vitamin D, RECORD): a randomised placebo-controlled trial. Lancet. 2005 May 7-13;365(9471):1621-8.

10. Gosch M, Kammerlander C, Roth T, Doshi HK, Gasser RW, Blauth M. Surgeons save bones: an algorithm for orthopedic surgeons managing secondary fracture prevention. Arch Orthop Trauma Surg. 2013 May 17 [Epub ahead of print]. 